\title{
First Determination of 2D Speed Distribution within the Bodies of Coronal Mass Ejections with Cross-correlation Analysis
}

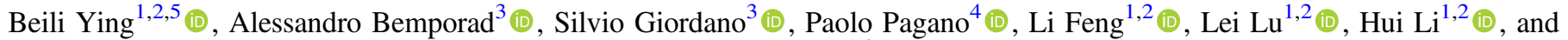 \\ Weiqun $\mathrm{Gan}^{1,2}$ \\ ${ }^{1}$ Key Laboratory of Dark Matter and Space Astronomy, Purple Mountain Observatory, Chinese Academy of Sciences, 210032 Nanjing, People's Republic of China \\ yingbl@pmo.ac.cn, lfeng@pmo.ac.cn \\ ${ }^{2}$ School of Astronomy and Space Science, University of Science and Technology of China, Hefei, Anhui 230026, People's Republic of China \\ ${ }^{3}$ INAF-Turin Astrophysical Observatory, via Osservatorio 20, I-10025 Pino Torinese (TO), Italy \\ ${ }^{4}$ School of Mathematics and Statistics, University of St. Andrews, North Haugh, St. Andrews, Fife, KY16 9SS, UK \\ Received 2019 March 29; revised 2019 May 27; accepted 2019 June 3; published 2019 July 23
}

\begin{abstract}
The determination of the speed of coronal mass ejections (CMEs) is usually done by tracking brighter features (such as the CME front and core) in visible light (VL) coronagraphic images and by deriving unidimensional profiles of the CME speed as a function of altitude or time. Nevertheless, CMEs are usually characterized by the presence of significant density inhomogeneities propagating outward with different radial and latitudinal projected speeds, resulting in a complex evolution eventually forming the interplanetary CME. In this work, we demonstrate for the first time how coronagraphic image sequences can be analyzed with the cross-correlation technique to derive two-dimensional (2D) maps of the almost instantaneous plasma speed distribution within the body of CMEs. The technique is first tested with the analysis of synthetic data and then applied to real observations. Results from this work allow us to characterize the distribution and time evolution of kinetic energy inside CMEs, as well as the mechanical energy (combined with the kinetic and potential energy) partition between the core and front of the CME. In the future, CMEs will be observed by two channels (VL and UV Ly $\alpha$ ) coronagraphs, such as Metis on board ESA Solar Orbiter mission as well as the Ly $\alpha$ Solar Telescope on board the Chinese Advanced Space-based Solar Observatory mission. Our results will help in the analysis of these future observations, helping in particular to take into account the 2D distribution of Ly $\alpha$ Doppler dimming effect.
\end{abstract}

Key words: magnetohydrodynamics (MHD) - methods: data analysis - Sun: coronal mass ejections (CMEs) - Sun: UV radiation - techniques: polarimetric

\section{Introduction}

Coronal mass ejections (CMEs) are large-scale phenomena related to huge magnetic plasma explosions from the Sun. With high speeds even up to $3500 \mathrm{~km} \mathrm{~s}^{-1}$ (Gopalswamy et al. 2009), these eruptions can release a large amount of energy $\left(10^{29}-10^{32} \mathrm{erg}\right)$ and plasma $\left(10^{15}-10^{16} \mathrm{~g}\right)$ ejected into the interplanetary space. When the accelerated plasma and the energetic particles propagate toward the Earth, geomagnetic storms can be caused.

In recent decades, many different instruments have been designed to observe different layers of the Sun and to reveal different physical phenomena, such as space-based visible light (VL) coronagraphs that are able to monitor the lower corona $24 \mathrm{hr}$ per day. The Large Angle Spectroscopic Coronagraph (LASCO; Brueckner et al. 1995) on board the Solar and Heliospheric Observatory (SOHO) mission successfully demonstrated that these coronagraphic instruments are capable of providing realtime information on the properties of solar eruptions, such as kinematics, masses, evolution of CMEs, and other parameters. The COR1 and COR2 coronagraphs on board the Solar TErrestrial RElations Observatory (STEREO) mission (Kaiser et al. 2008) have a big advantage in observing the propagation of CMEs simultaneously from two different perspectives, thanks to the STEREO twin spacecraft. These coronagraphs have offered the best way to investigate the geometrical structure of CMEs. Many methods to reconstruct CMEs' three-dimensional (3D)

\footnotetext{
5 This work was mainly carried out at INAF-Turin Astrophysical Observatory, via Osservatorio 20, I-10025 Pino Torinese (TO), Italy.
}

geometrical structure have been developed, such as mask fitting (Feng et al. 2012), forward modeling, triangulation method, polarization ratio method ( $\mathrm{Lu}$ et al. 2017), and the graduated cylindrical shell model (Thernisien et al. 2006, 2009).

The UV Coronagraph Spectrometer (UVCS; Kohl et al. 1995) on board $\mathrm{SOHO}$ also allowed us to investigate in great detail the CME events by using the UV emission lines (such as H I Ly $\alpha 121.6 \mathrm{~nm}$, O VI 103.2, and O VI $103.7 \mathrm{~nm}$ spectral lines), but its field of view (FOV) was limited to the spectrometer entrance slit $\left(42^{\prime}\right)$. Observations from UVCS have been used to derive plasma proton and electron temperatures, heavy ion kinetic temperatures, and elemental distributions (e.g., Akmal et al. 2001; Ciaravella et al. 2003, 2006; Bemporad et al. 2007, 2018; Susino \& Bemporad 2016), while all of this information cannot be obtained from VL coronagraphs alone. Unfortunately, after almost $17 \mathrm{yr}$ of successful observations of CMEs, solar wind, and other phenomena, the UVCS instrument has not been operational since 2012. Many of the previous studies based on the analysis of VL coronagraphic observations concentrate on the estimation of CME masses and subsequently on deriving the kinetic and potential energies (e.g., Vourlidas et al. 2000; Colaninno \& Vourlidas 2009; Carley et al. 2012; Feng et al. 2013). Nevertheless, when researchers estimate the kinetic energy of a CME, they always use the unidimensional speed measured for the front or the core of the CME and ignore its anisotropic kinematical properties.

Despite the importance of this topic, the two-dimensional (2D) distribution of the kinematical properties of CME plasma 
was studied in only a few previous works, and with many different techniques. Low \& Hundhausen (1987) calculated the velocity field of a CME based on visual tracking of features in subsequent images. Tappin et al. (1999) calculated the outflow speeds of the solar wind by using a cross-correlation method, but they did not apply this method to CMEs. Robbrecht \& Berghmans (2004) used the modified Hough transform to filter the most significant signals of CMEs and calculated their average speed while regarding the CMEs as bright ridges in (time, height) maps. They did not generate a velocity field over the whole CME. Colaninno \& Vourlidas (2006) used optical flow methods to estimate the velocity fields of whole CME bodies. Feng et al. (2015a) provided a method to investigate the CME kinematics via determining the radial mass transport process throughout the entire $\mathrm{CME}$, which is capable of estimating the radial flow speed inside the CME. Braga et al. (2017) used the CORSET3D to determine the tie points along the CME front automatically in 3D space and derived the average 2D speed of CME fronts in the Stonyhurst coordinate system. Compared with these studies, all of these results have never shown the 2D kinetic and potential energy distributions of CMEs.

In this paper, we successfully use the VL coronagraphic images to obtain the 2D speed map of the plasma within the body of a CME using the cross-correlation method and to derive the first kinetic energy distribution of the CME. In the next few years, new coronagraphic instruments with new channels will be launched into space. The Metis instrument (Antonucci et al. 2017) on board the ESA Solar Orbiter mission (to be launched in 2020) will provide the first ever simultaneous observations of the solar corona with FOV of $1.6-2.9$ (the corresponding projected altitudes in solar radii from 1.7 to $3.1 R_{\odot}$ when the spacecraft will be at closest approach at $0.28 \mathrm{au}$ ), combining two different spectral bands: broad band (580-640 nm) in the VL and narrow band in the UV emission from the neutral $\mathrm{H}$ I atoms (121.6 nm Ly $\alpha$ line, the most intense line in the UV solar spectrum). Similar to Metis, the LST instrument (Ly $\alpha$ Solar Telescope; Li 2015; Li et al. 2019; Feng et al. 2019) on board the future Chinese Advanced Space-based Solar Observatory (ASO-S) mission (Gan et al. 2015, to be launched in 2022) will also observe the corona in the VL and in the UV H I Ly $\alpha$ line, with FOVs from solar disk to $1.2 R_{\odot}$ and $1.1 R_{\odot}$ to $2.5 R_{\odot}$. As we will discuss, the CME analysis technique being presented here will be very important to support future observations of solar eruptions that will be acquired by these instruments.

The mechanism of formation of the $\mathrm{HI} \operatorname{Ly} \alpha$ line from an optically thin corona is a combination of radiative and collisional excitations. The radiative component is generated by resonant scattering of the chromospheric and photospheric radiation by coronal neutral $\mathrm{HI}$ atoms, while the collisional component is due to the de-excitation of a coronal atom previously excited by collision with a free electron. When the CME moves outward from the Sun, the intensity of the H I Ly $\alpha$ line due to radiative excitation will decrease due to the Doppler dimming effect. According to Noci et al. (1987), the Doppler dimming factor is defined as follows:

$$
F_{D}\left(v_{r}\right)=\int_{0}^{+\infty} I_{\mathrm{ex}}(\lambda-\delta \lambda) \Phi\left(\lambda-\lambda_{0}\right) d \lambda,
$$

where $I_{\mathrm{ex}}(\lambda-\delta \lambda)$ is the intensity line profile of the chromospheric radiation. $\delta \lambda=\frac{\lambda_{0}}{c} v_{r}$ is the wavelength shift of the exciting profile due to the radial velocity of the moving plasma $v_{r}$, and $c$ is the light speed. The term $\Phi\left(\lambda-\lambda_{0}\right)$ is the normalized coronal absorption profile along the direction of the incident radiation, and $\lambda_{0}$ is the reference wavelength of the line transition. If the Doppler dimming factor can be estimated in advance, we could be able to obtain the electron temperature by the combination of the VL images and the UV H I Ly $\alpha$ intensity observations. Without spectroscopic observations, our method to derive the velocity distribution of the CME can be considered a useful diagnostic technique to estimate the 2D distribution of Doppler dimming factors of the observed CME in the future data.

Susino \& Bemporad (2016) first combined polarized VL images from LASCO/C2 and simultaneous data in the UV H I Ly $\alpha$ line $(1216 \AA$ ) acquired by the UVCS spectrometer to estimate the CME plasma electron temperature, given the CME speed measured from LASCO images. The purpose of that work was to test the possible determination of $\mathrm{CME}$ plasma temperatures from the analysis of VL images and UV H I Ly $\alpha$ intensities, without spectroscopic data. Subsequently, Bemporad et al. (2018) has rebuilt the synthetic images of total brightness (tB), polarized brightness $(\mathrm{pB})$, and H I Ly $\alpha$ intensities by using the output parameters from a numerical 3D MHD simulation of a CME. These synthetic images have been analyzed to measure the CME plasma temperatures, constrained by the simulated parameters, while taking into account the Doppler dimming effect. In our paper, we calculate the velocity distribution of simulated CME (on the basis of the method described in Bemporad et al. 2018) and apply the same technique to a real event.

In particular, in this paper, we first of all, estimate the measurement error of the speed obtained from our method by using the MHD simulated results in Section 2. Then we apply the cross-correlation method to a real CME event and obtain the first 2D speed and the Doppler dimming factor distribution of the CME in Section 3, as well as the first kinetic energy distribution in a 2D map. Moreover, we also derive the partition and evolution of the mechanical energy, combined with the kinetic and potential energies, between the core and front of the CME. Discussions and conclusions are described in Section 4.

\section{Measurement of the Radial Speed of the CME from Synthetic VL Images}

In the first part of this work we generate synthetic VL images of a CME using the same parameters from the MHD simulation of Pagano et al. (2014). We refer to this work for details. The advantage of using VL synthetic images to test the technique for radial speed determination is that in these images the measured intensities are independent of the plasma outflow speed, which is different from what happens in the UV H I Ly $\alpha$ images. Here we compute the VL tB from each simulated plasma element by integrating the contribution along a given line of sight (LOS) and then obtain the synthetic tB images. These have been derived from the computation of the Thomson-scattered light from a single electron in each cell, multiplied by the electron number density of each cell, as the Thomson-scattered light only depends on the position with respect to the solar disk and the scattering angle (Billings 1966). 

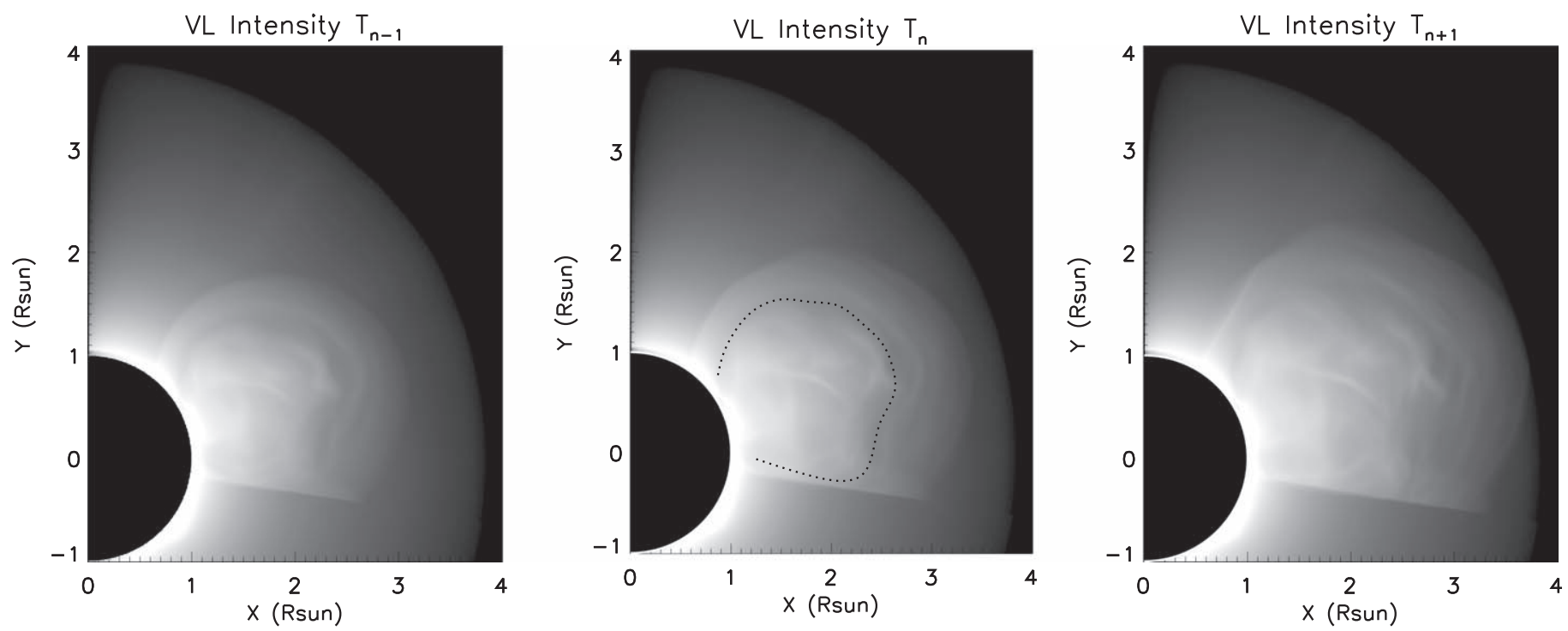

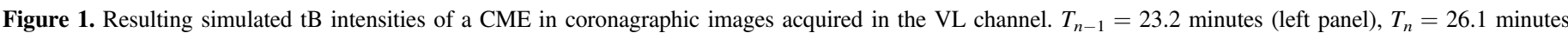

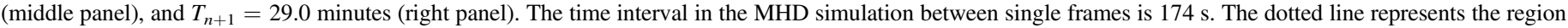
of the CME core.

\subsection{Method}

The resulting sequence of VL coronagraphic intensity images is shown in Figure 1. These three synthetic VL images are at three simulated times $T_{n-1}=23.2, T_{n}=26.1, T_{n+1}=$ 29.0 minutes, respectively. We use these three VL images (at $T_{n-1}, T_{n}, T_{n+1}$ times) of the CME to measure its radial velocity at the $T_{n}$ time. Details of the method are listed: first, we use the normalized running-difference image (normalized by the previous image) to enhance the faint structures of the CME through a radial filter convolving with a Gaussian kernel (the SolarSoft routine filter_image.pro). Second, in order to measure the radial component of the plasma speed, we convert each VL filtered image from Cartesian to polar coordinates. This step allows us to extract the radial VL intensities at the three times at each fixed position angle, the angle going from $0^{\circ}$ to $90^{\circ}$ and measured from the equatorial plane (corresponding to the east-west direction). Then the radial intensity distributions at three different times are analyzed in three steps: forward step (FS), backward step (BS), and average step (AS). In the FS, we determine pixel by pixel the radial shift by maximizing the cross-correlation between the signal in the actual frame (at $T_{n}$ ) extracted in a symmetric radial window centered on the considered point and the signal in a shifted radial window extracted in the next frame (at $\left.T_{n+1}\right)$. This first step is analogous to the method described in Bemporad et al. (2018) but is improved here as we are going to describe. According to the assumption that the CME plasma is expanding faster in the front with respect to the core, we assume that the size of the shifted radial window increases linearly with the radial distance in the frame at $T_{n+1}$, so that we can obtain more corresponding signals, while the window at $T_{n}$ has constant size moving forward. Then the offset between the considered point and the central point of the matched window was regarded as the correspondent displacement. Subsequently, given the time interval between each frame (174s), the displacement can be converted to the radial speed at the considered point. The BS analysis is similar, but with two differences with respect to the FS: (1) in the BS, we determine the maximal value of cross-correlation between the frame at the
$T_{n}$ and the frame at the $T_{n-1} ;(2)$ the shifted radial window at $T_{n-1}$ is shrinking linearly, while the window size at $T_{n}$ moment is fixed. Finally, the average speed value obtained from FS and $\mathrm{BS}$ is considered the AS. Figure 2 shows the $2 \mathrm{D}$ radial velocity images as obtained with these three steps. From Figure 2 it is clear that overall the speed maximizes at the CME front and decreases at lower heliocentric distances, as expected. Moreover, the plasma speed distribution within the CME body displays significant spatial inhomogeneities, due to the fact that different parts of the CME plasma are propagating with different speeds and accelerations.

\subsection{Uncertainties in CME Radial Velocity}

In order to discuss the results from our method, we first choose two input velocities ( $t=26.1$ minutes) obtained from the MHD simulation as reference velocities. In fact, due to LOS integration, the result of any kind of analysis applied to 2D coronagraphic images will be a $2 \mathrm{D}$ velocity map, while the MHD simulation provides us with $3 \mathrm{D}$ data cubes. Here, following the analysis performed by Bemporad et al. (2018) for the optimization of plasma temperature determination in CMEs, we consider two reference velocities. The first one is simply the $2 \mathrm{D}$ cut of the radial velocity data cube in the plane of sky (POS). The second one is the average LOS velocity weighted over the electron number density $v_{\mathrm{LOS}}=$ $\int_{-\infty}^{+\infty} n_{e} v_{r} d z / \int_{-\infty}^{+\infty} n_{e} d z$, considering that the VL brightness is proportional to the electron number density, $n_{e}$, of each cell, where $n_{e}=\rho /\left(0.83 m_{p}\right)$ and $m_{p}$ is the proton mass. Here we assume that the typical coronal plasma condition of fully ionized atoms with $\mathrm{He}$ abundance is equal to $10 \%$. These two different velocity distributions are shown in Figure 3 with the same color scale for comparison. It shows that significant differences between the two velocity distributions are present only around the nose of the CME front, where integration along the LOS is more significant. In the right panel of Figure 3, the speed distribution maximizes around the nose region, while this is not the case in the left panel.

The differences between the velocity maps measured from the synthetic VL images with the three methods (FS, BS, and 

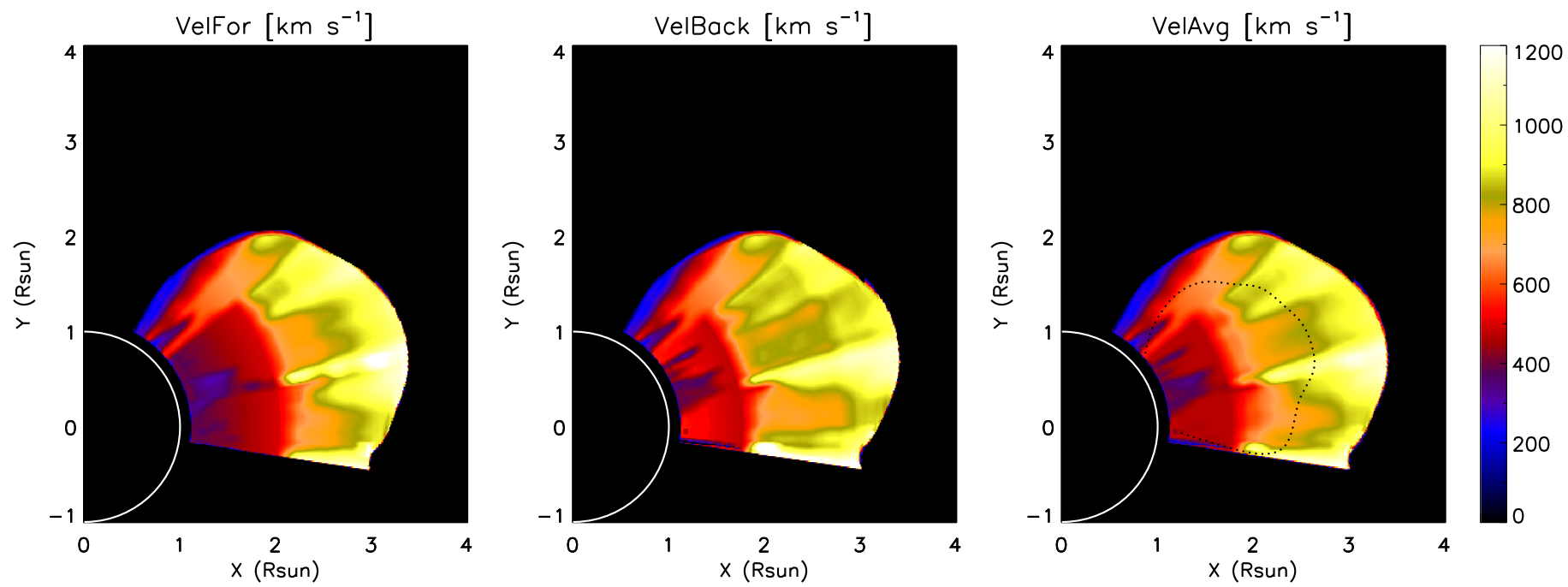

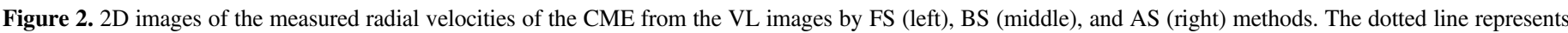
the region of the CME core.
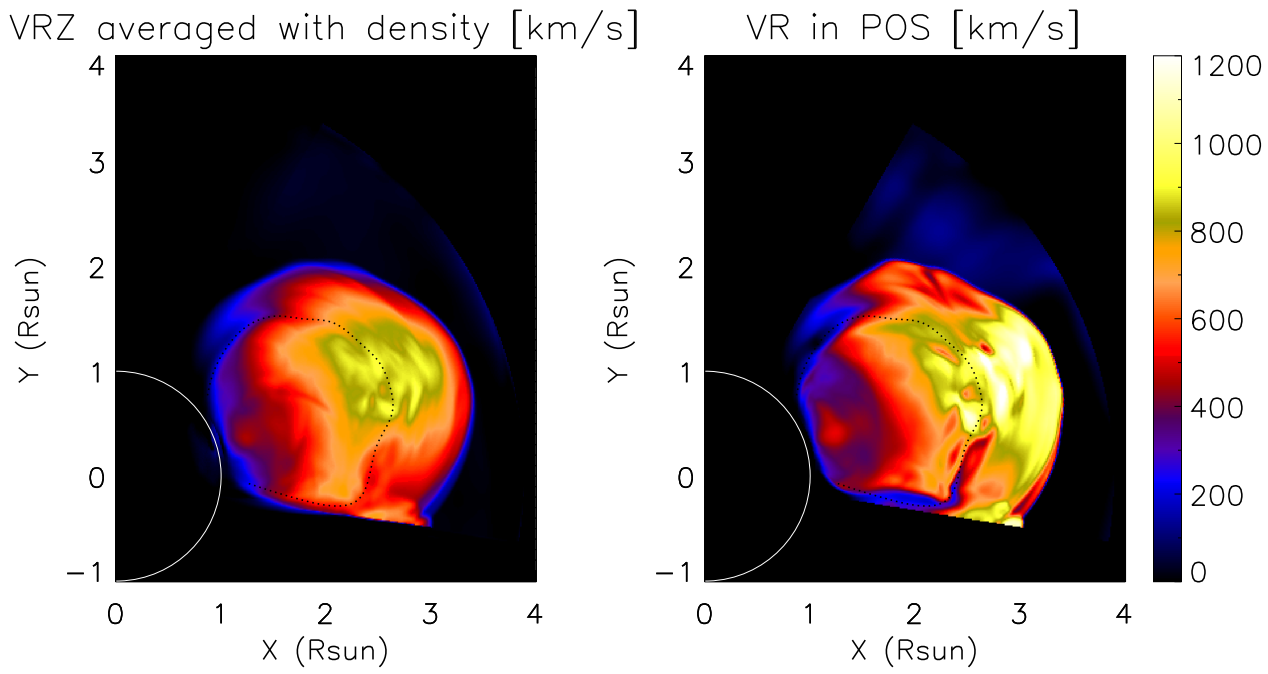

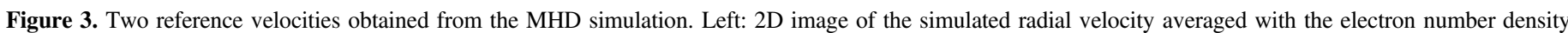

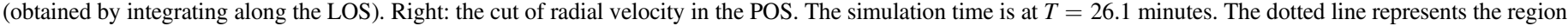
of the CME core.

AS) and the two reference velocities mentioned above, subsequently normalized to the reference velocities, are socalled relative comparisons, as shown in Figure 4. In particular, the top three panels in this figure represent the relative comparisons between the measured radial velocities (from the FS, BS, and AS, respectively) and the reference radial velocities averaged with the density, while the bottom panels show the relative comparisons between the measured velocities and the simulated velocities in the POS (FS, BS, and AS, respectively). Considering in particular the comparison between the two AS maps and reference velocities (Figure 4, right column), it turns out that the measured radial velocities conform better with respect to the reference speed weighted with the number density in the core of the CME (within $20 \%$ of uncertainty on average). On the other hand, the relative comparison with the POS speed (Figure 4, right bottom) shows a more uniform distribution, with a good velocity estimate (within $30 \%$ of uncertainty on average) of the plasma speed distribution in the POS all over the CME body, except for the flanks (where large errors up to about $100 \%$ are present). In general, we expect that the method described above provide us with a good uncertainty about the radial velocity distribution within the CME body, with the exception of the regions located near the flanks of the eruption.

\section{Analysis of a Real Event}

\subsection{Observations}

To test the techniques described above on a real event, we selected a CME that was observed by STEREO coronagraphs. They have better time cadences with respect to $\mathrm{SOHO}$ coronagraphs. We decided to focus on a limb event in order to minimize possible projection effects due to plasma expansion out of the POS. On 2010 October 28, a CME with a classical three-part structure started to erupt at around $\sim 10: 00$ UT. The source region of this eruption was located in active region (AR) NOAA 11115, where a transient flare occurred. The CME, rising up from a streamer, was then observed above 

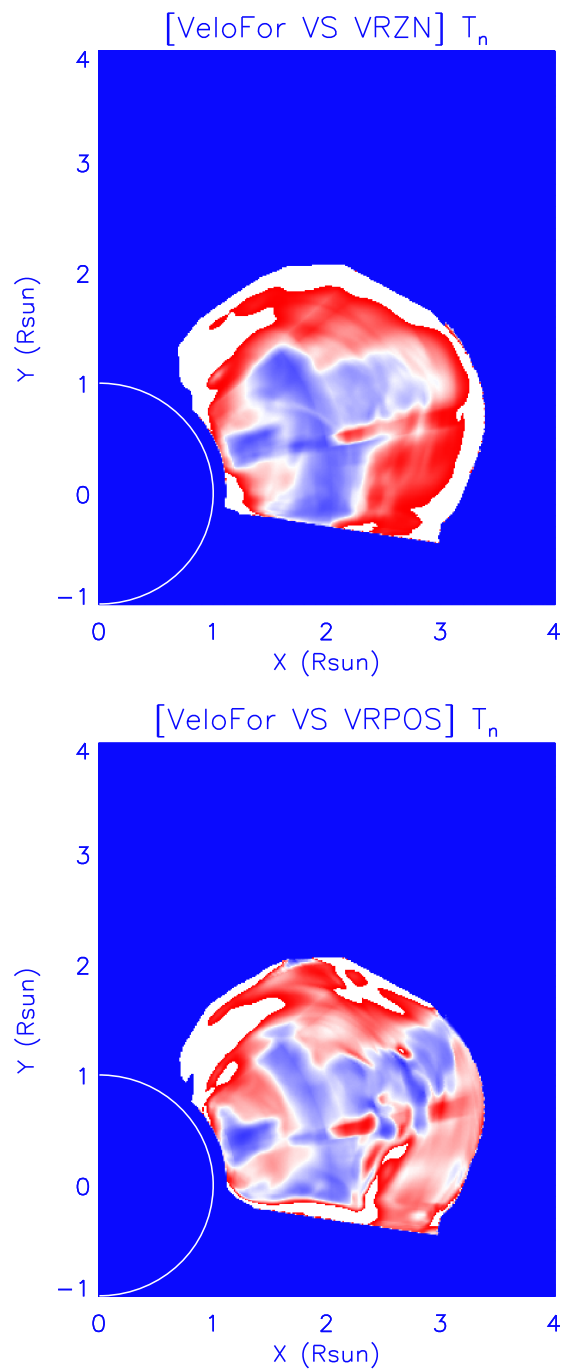
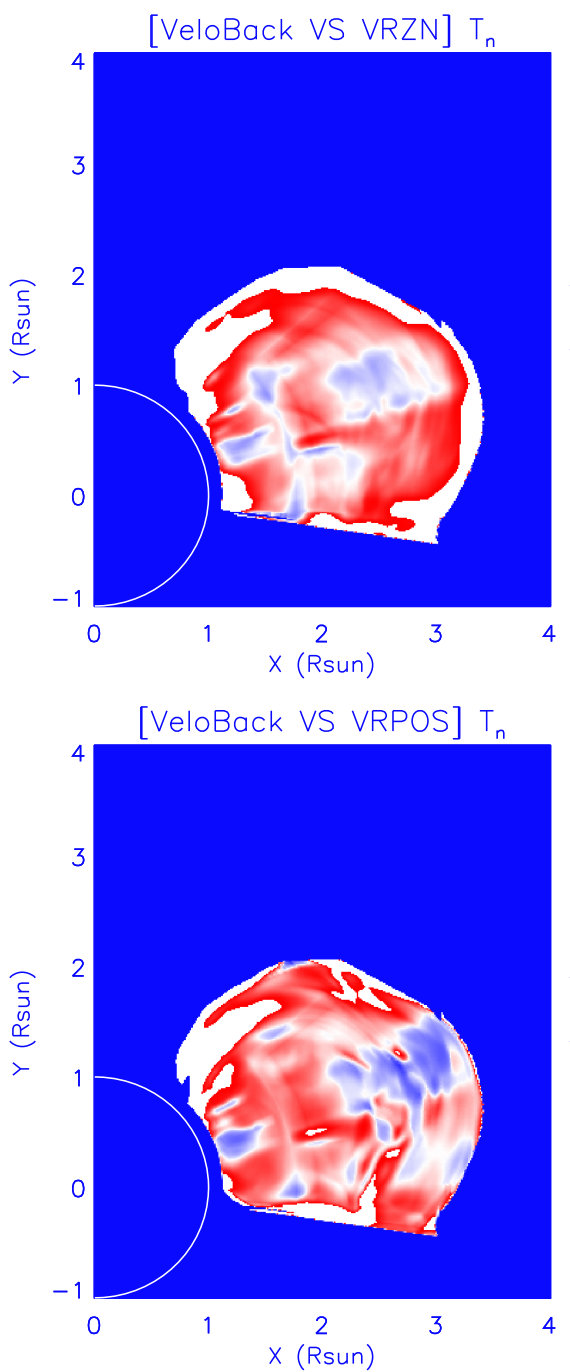
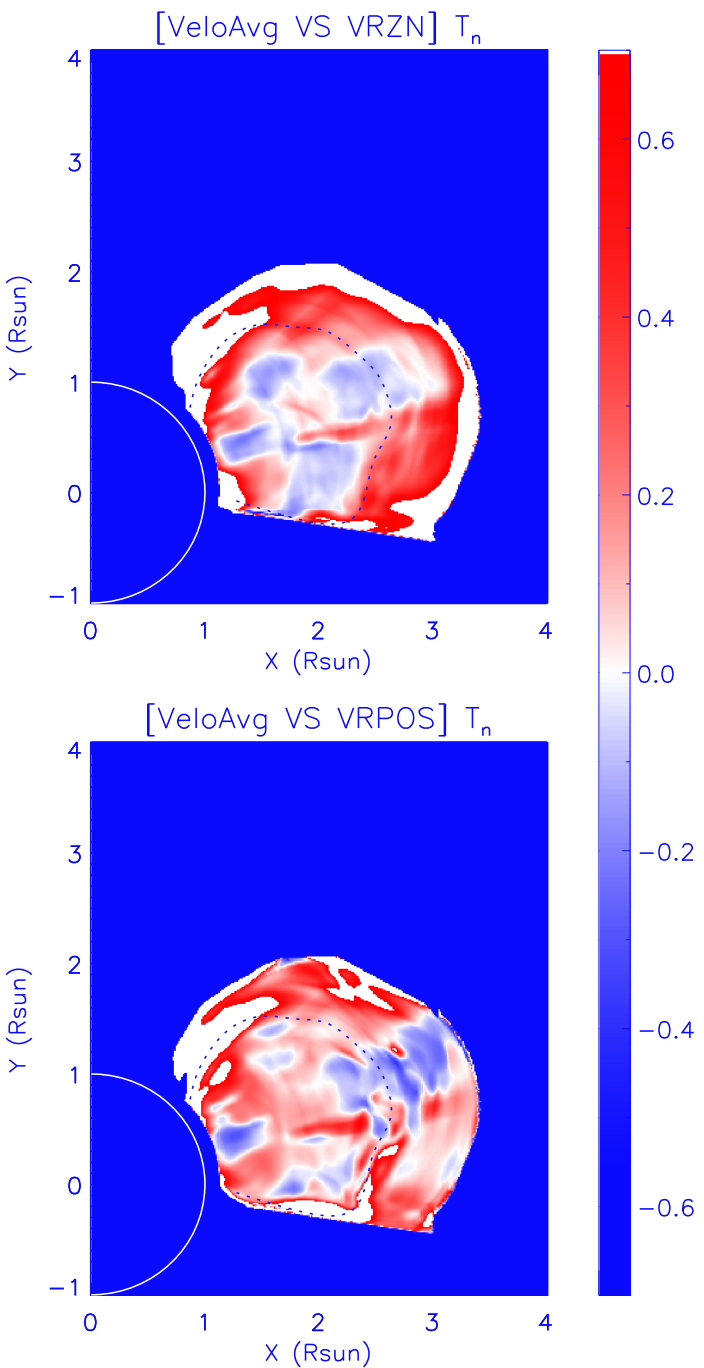

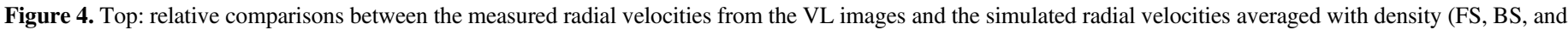

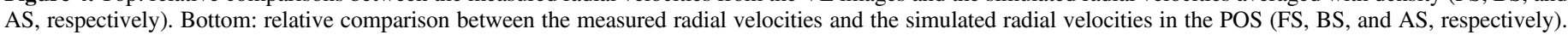
The dotted lines represent the region of the CME core.
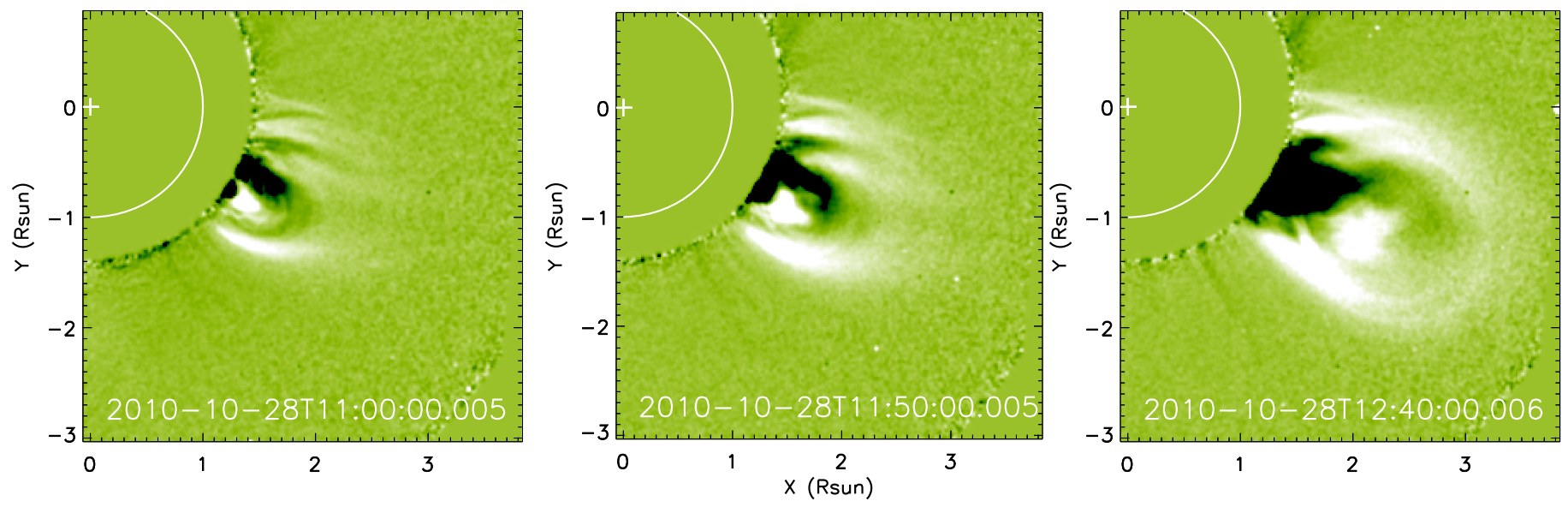

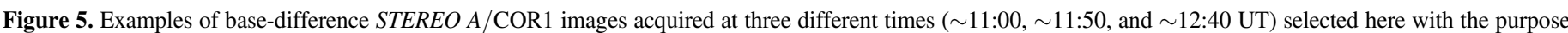

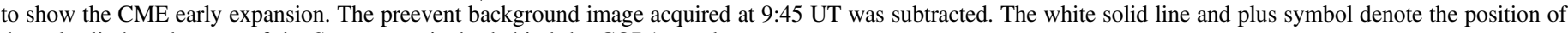
the solar limb and center of the Sun, respectively, behind the COR1 occulter.

the solar limb in the FOV of both the COR1 and COR2 coronagraphs on board the STEREO. COR1 images reach a lower heliocentric distance, with an FOV going from 1.4 to
$4 R_{\odot}$, while COR2 FOV goes from 2.5 to $15 R_{\odot}$. We fitted the graduated cylindrical shell model (Thernisien et al. 2009) to the CME observed by COR1 A and B and find that the propagation 

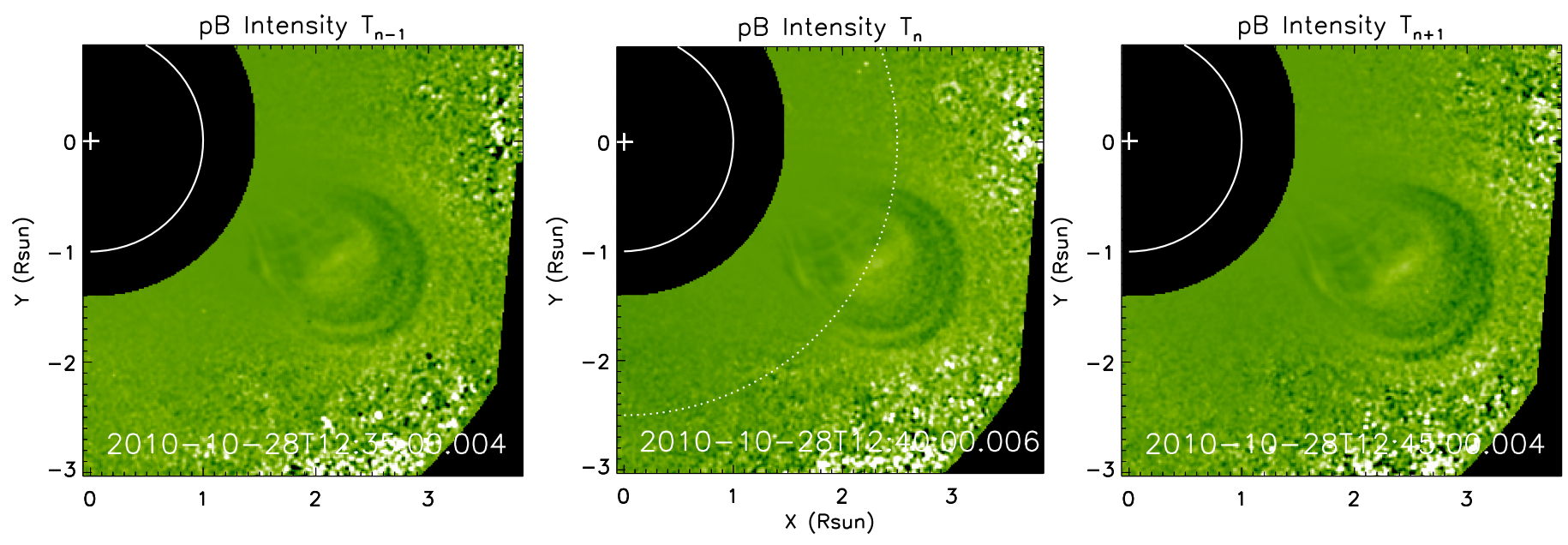

Figure 6. STEREO A/COR1 normalized running-difference images (acquired at 12:35, $\sim 12: 40$, and $\sim 12: 45$ UT). The position of a dotted line is at $r=2.5 R_{\odot}$.

of the CME deviates from the POS with an angle of about $10^{\circ}$ as seen from STEREO A. Therefore, we assume that this limb CME propagates approximately in the POS of STEREO A.

In order to show the CME early expansion, three $\mathrm{pB}$ images of the CME acquired at three different times are shown in Figure 5. All images were processed by standard SolarSoft routines secchi_prep.pro. To better show the CME, these images are subtracted by the preevent background image at around 09:45 UT; hence Figure 5 shows the evolution of the $\mathrm{CME}$ excess brightness. The dark region in the core part of the $\mathrm{CME}$ is due to the presence of a streamer in the preevent images. At the initial stage, the evolution of the CME shows only a slow change of local dynamics in the streamer. Then, with the acceleration phase, the CME structure separates from the streamer, evolving into a typical bubble shape.

\subsection{D Maps of the CME Velocities}

To test the velocity measurement technique described in Section 2.1, we selected here only three subsequent images observed from STEREO A/COR1 at three different times. The advantage of the STEREO COR1 data (compared with other coronagraphs) is that their high temporal resolution of 5 minutes is close to the time interval of the simulated VL image. We use the normalized running-difference images and subsequently enhance the contrast of the CME structure to measure the radial velocities of the $\mathrm{CME}$ in each pixel. As shown in Figure 6, many detailed structures in the CME body can be observed in the resulting images. We mark these three sequential images (acquired at $\sim 12: 35, \sim 12: 40, \sim 12: 45$ UT) as $T_{n-1}, T_{n}$, and $T_{n+1}$ times, respectively. Through the three steps (FS, BS, and AS) of our method, the radial velocity maps have been derived, which are shown in Figure 7 (top row). It turns out that around 12:40 UT the speed of the CME body is not larger than $400 \mathrm{~km} \mathrm{~s}^{-1}$, and the front speed is higher than the core, as expected. Moreover, the radial speed also maximizes around the northward flank of the CME, suggesting a significant latitudinal asymmetry in the CME propagation. The interpretation of this asymmetry is shown in Figure 8: while the source AR was located at a latitude of $29^{\circ} \mathrm{S}$, the nose of the CME front (representing the most likely $\mathrm{CME}$ propagation direction) was centered around $\sim 15^{\circ} \mathrm{S}$. The CME was deflected northward with respect to the source AR. Comparing the shape of the CME front between that observed at 12:40 UT (Figure 5, right panel) and at 14:54 UT (Figure 8), it is almost circular at 12:40 UT, while that is not the case in the FOV of COR2. The front in the northward part of the CME seems to be moved further, and this is well reproduced by the faster radial speed we derived in the $2 \mathrm{D}$ velocity distribution.

Given the 2D velocity maps, asymmetries and inhomogeneities in the $2 \mathrm{D}$ velocity distribution can be better explored by comparing these $2 \mathrm{D}$ velocities with the front speed measured along the propagation direction of the CME nose. In fact, this latter quantity is usually assumed in the literature to be representative of the speed in the whole CME body. The CME nose speed here was derived by extracting a radial slice in the excess brightness images to track its propagation along the nose direction; the resulting nose speed is about $\sim 320 \mathrm{~km} \mathrm{~s}^{-1}$. The bottom panels of Figure 7 show the differences between the $2 \mathrm{D}$ radial speed (Figure 7, top row) measured from the VL images in the CME body and the speed value measured at the CME nose. The comparison shows again that in some parts of the northward flank of the CME the radial plasma speed is even higher than the nose speed. The reasons for that are as follows: (1) as mentioned above, the CME was propagating northward with higher radial speed and hence a latitudinal north-south asymmetry was expected; and (2) as we showed with the analysis of synthetic data the uncertainties in the estimate of the CME velocity maximize at the flanks of the eruption. Moreover, as previously discussed by Bemporad et al. (2018), each packet of plasma in the 2D image, in principle, could have a different component of velocity along the LOS. However, this component cannot be measured through the analysis of coronagraphic images and can be estimated only with spectroscopic data (from the Doppler shift of spectral lines), as was done many times in the literature with the analysis of $\mathrm{SOHO}$ /UVCS observations of CMEs (see, e.g., Raymond et al. 2003; Lee et al. 2009).

As we are going to show, the 2D maps of CME radial velocity derived in the previous sections can be used to estimate the 2D distribution of further parameters that can be of interest. First of all, the velocity map can be employed to derive the 2D distribution of Doppler dimming factors for the UV H I Ly $\alpha$ intensity, a parameter that will be very important for the future analysis of CME coronagraphic observations acquired in this band (see Section 3.3). Moreover, these velocity maps can be used to investigate the $2 \mathrm{D}$ distribution and time evolution of 

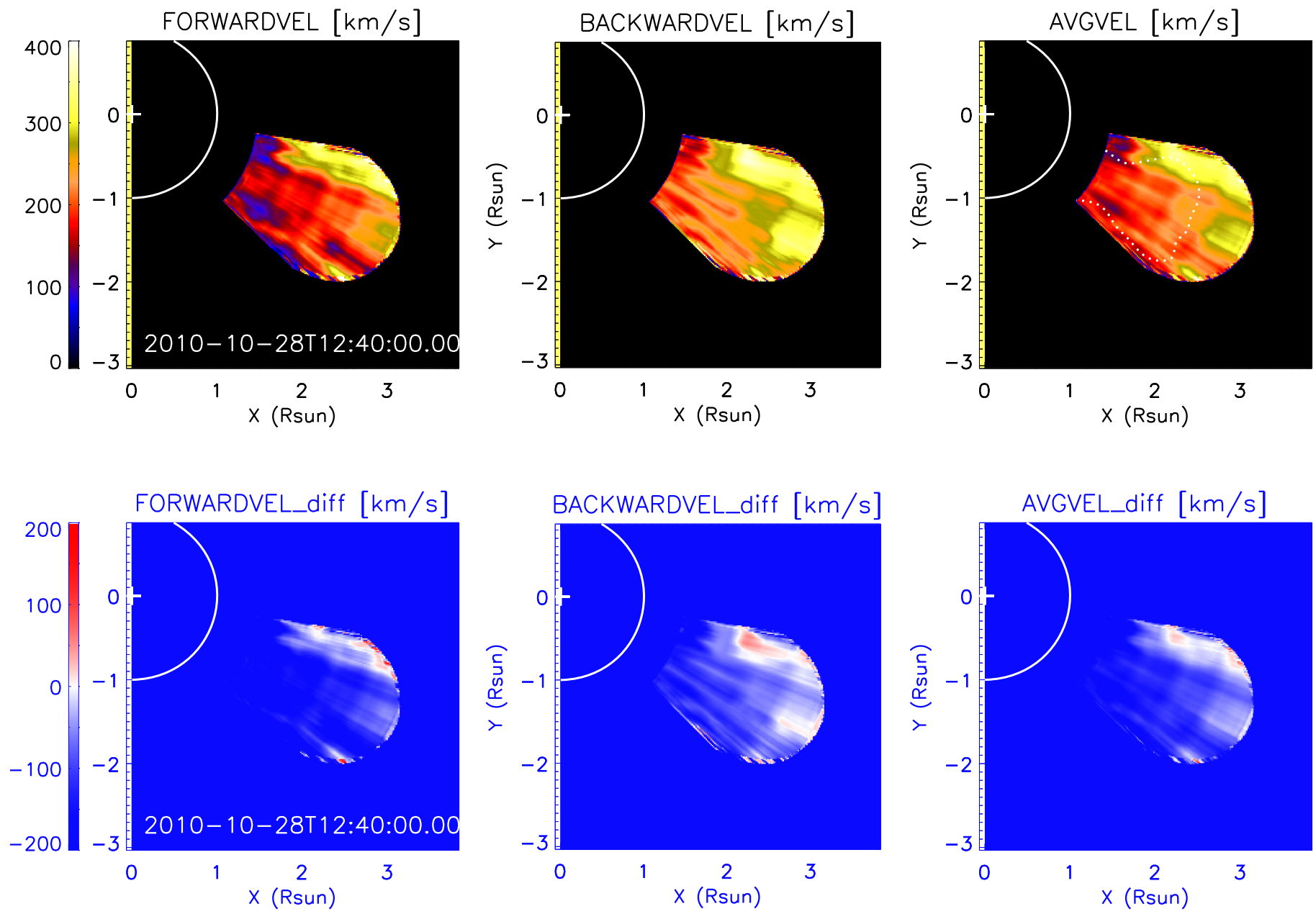

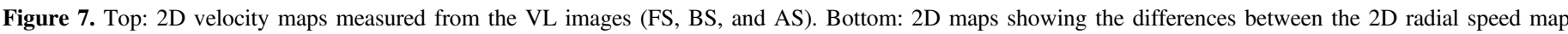

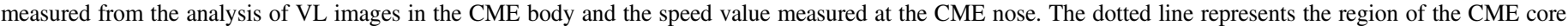
defined in Section 3.4 and shown in Figure 10.

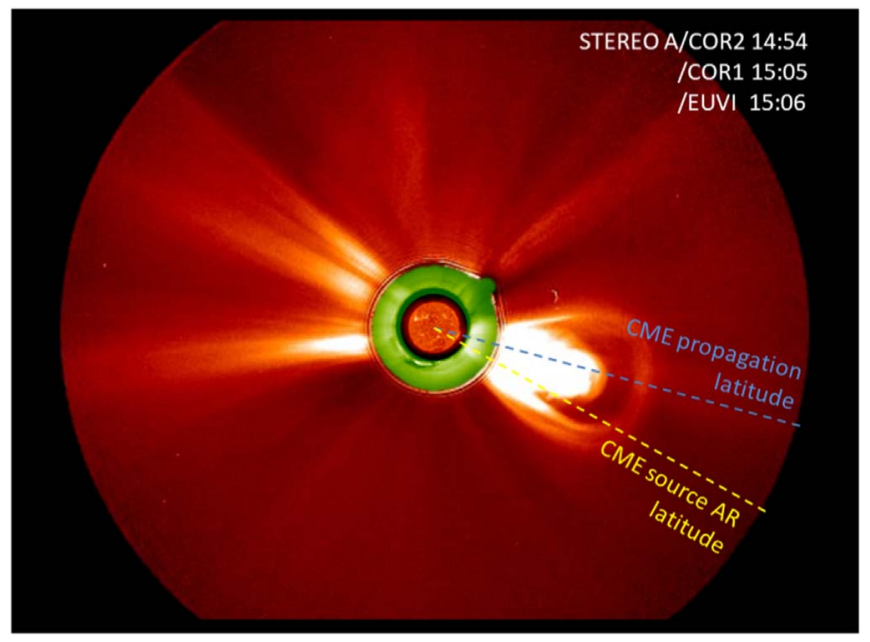

Figure 8. Superposition between STEREO A/EUVI, COR1, and COR2 images acquired on 2010 October 28 at 15:06, 15:05, and 14:54 UT, respectively. This figure shows the difference between the source AR latitude at $29^{\circ} \mathrm{S}$ (yellow dashed line) and the $\mathrm{CME}$ propagation latitude around $\sim 15^{\circ} \mathrm{S}$ (cyan dashed line).

the plasma kinetic energy in the CME body, which can provide interesting information on the energetic of the CME (see Section 3.4).

\subsection{Doppler Dimming Factor}

In the UV spectral range, the primary physical processes responsible for atomic excitation (followed by spontaneous emission) are the collisional excitation and the photoexcitation. These are also the main contributions of the H I Ly $\alpha$ emission in the optically thin corona, while other mechanisms could be neglected, such as the Thomson scattering of chromospheric radiation by free coronal electrons. The observations from the coronagraphs only provide 2D images; according to Susino \& Bemporad (2016), the radiative component of the H I Ly $\alpha$ can be expressed as

$$
\begin{gathered}
N_{e} \equiv \int_{\mathrm{LOS}} n_{e} d z \approx\left\langle n_{e}\right\rangle L, \\
I_{\mathrm{rad}} \propto R\left(T_{e}\right) \cdot F_{D}\left(v_{r}\right)\left\langle n_{e}\right\rangle L,
\end{gathered}
$$

where $N_{e}$ is the column density that can be obtained from VL images, $\left\langle n_{e}\right\rangle$ is the average electron density of the CME plasma along the LOS, and $L$ is the thickness of the CME plasma along the LOS. If the thickness $L$ of emitting plasma is known, the column density $N_{e}$ can be converted to volume density $\left\langle n_{e}\right\rangle$ in units of $\mathrm{cm}^{-3}$. In Equation (3), $R\left(T_{e}\right)$ is the element ionization fraction, which is a function of the electron temperature $T_{e}$, and $F_{D}\left(v_{r}\right)$ is the Doppler dimming factor, as defined in 

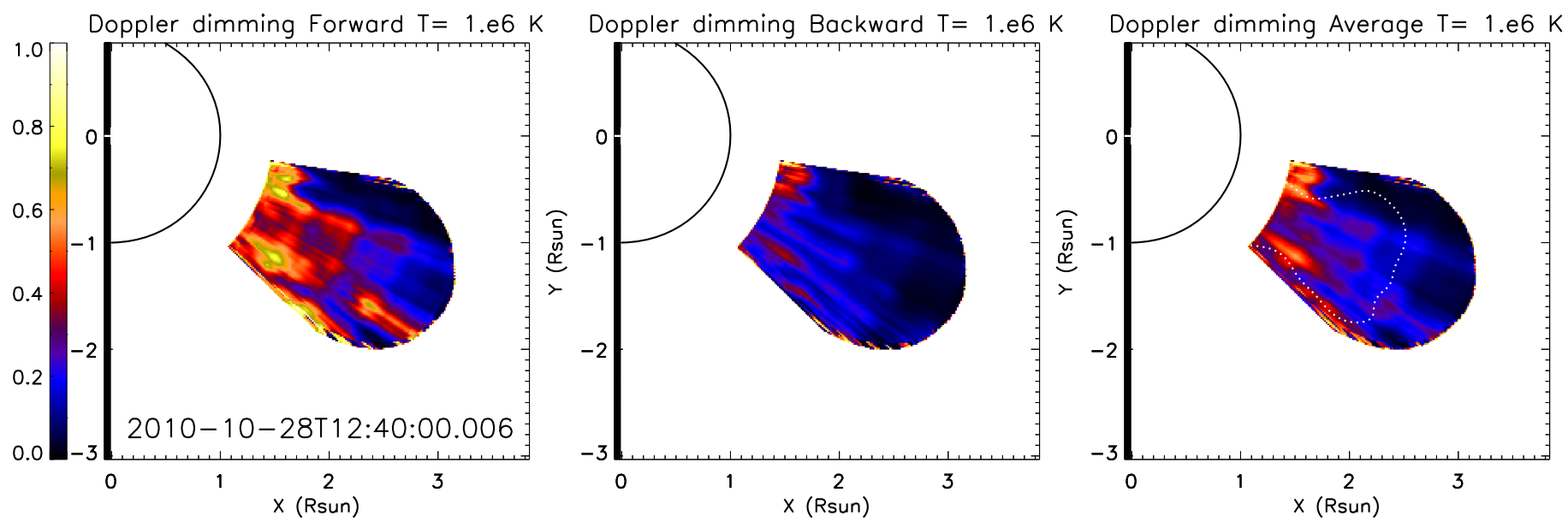

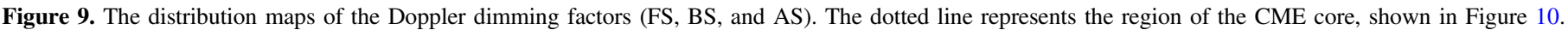

Equation (1). In the future, if the Doppler dimming factor and the thickness of the plasma along LOS can be determined, combining the observations of VL and UV intensities, we could estimate the temperature distribution of the CME plasma, according to Equation (3). For instance, with a similar analysis (based only on VL and UV H I Ly $\alpha$ intensities), Susino \& Bemporad (2016) recently found that CME cores are usually associated with cooler plasma $\left(T \sim 10^{6} \mathrm{~K}\right)$ and that a significant increase of the electron temperatures is observed from the core to the front of the CME (where $T>10^{6.3} \mathrm{~K}$ ).

However, in the analysis described above, the Doppler dimming factor will be affected not only by the radial velocity of the CME plasma, but also by the shape of normalized coronal absorption profile, $\Phi(\lambda)$, and by the intensity spectrum of incident profile, $I_{\mathrm{ex}}$, as described by Equation (1). The normalized coronal absorption profile here has been assumed to be Gaussian with a width $\sigma_{\lambda}$, under the assumption of a Maxwellian velocity distribution of the absorbing particles, where

$$
\sigma_{\lambda}=\frac{\lambda_{0}}{c} \sqrt{\frac{k_{\mathrm{B}} T_{k}}{m_{p}}}(\mathrm{~cm}) .
$$

In Equation (4), $k_{\mathrm{B}}$ is the Boltzmann constant and $T_{k}$ is the kinetic temperature. To calculate the $F_{D}$, we assumed here an averaged value of the exciting radiation coming from the solar disk and adopted the $\mathrm{SOHO} /$ solar ultraviolet measurement of the emitted radiation chromospheric spectral profile observed at solar minimum in 1996 July (Lemaire et al. 2002). The kinetic temperature is assumed to be $T_{k}=10^{6} \mathrm{~K}$ for the normalized coronal absorption profile. The resulting $2 \mathrm{D}$ distribution of the Doppler dimming factors is shown in Figure 9. Due to the low speed of the CME body, the distribution of the $F_{D}$ factors reflects the anisotropies in the 2D distribution of CME velocity, while $F_{D}$ decreases sharply in the pixels where the CME plasma is expanding faster than $\sim 300 \mathrm{~km} \mathrm{~s}^{-1}$. The resulting 2D distribution of the $F_{D}$ factors further confirms that (as recently shown by Bemporad et al. 2018) the UV intensity in the CME front will be severely attenuated due to the larger plasma radial speed, as can also be revealed by comparing the distribution of the Doppler dimming factors in Figure 9 with the radial speed distribution of the CME in Figure 7. In the future, when we estimate the electron temperature of CMEs propagating with a very high speed, it will even be possible to neglect the contribution of the radiative component and to consider only the collisional component of H I Ly $\alpha$ intensity.

\subsection{Energies of the CME}

To estimate the CME mechanical energy (including the potential and the kinetic energies), the first quantity that we need to estimate is the CME mass. There are different ways to achieve this, for example, by using EUV dimming and spectroscopic observations and, the most common way, from Thomson scattering using VL coronagraphic images (e.g., Aschwanden et al. 2009; Colaninno \& Vourlidas 2009; Carley et al. 2012; Tian et al. 2012; Feng et al. 2013; Aschwanden 2016). In this work, the Thomson-scattering approach is applied and the STEREO/COR1 pB images were used. Many previous investigators (Mierla et al. 2011; Howard 2015a, 2015b) have shown that in COR1 observations of CMEs the Thomson-scattered signal will be strongly contaminated by $\mathrm{H} \alpha$ emission from the prominence, subsequently resulting in the overestimation of the CME mass. Here we emphasize that in our event there is no filament structure that erupted and propagated into the FOV of COR1 instrument, thus the contribution of $\mathrm{H} \alpha$ emission can be ignored and the mass measured from COR1 can be considered reliable.

The major part of the signal of white light coronagraph images, yielded by Thomson scattering, is linked linearly to the density of the scattering free electrons. The major non-Thomson-scattered contributions, like stray light and dust scattering (the F-corona), are usually removed by subtracting the preevent background image. The remaining image brightness then should contain the CME intensity distribution produced by Thomson scattering alone by electrons in the CME plasma. In our event, however, before the appearance of the CME, there exists a dense streamer for a long time, and subtracting the preevent background image (Figure 10, top left), which also includes the streamer structure, will result in negative values appearing in many pixels of the difference image (Figure 10, top right), causing a large underestimate of the CME mass. Therefore, here we consider the image resulting from the subtraction of the preevent image as the lower limit estimate of the CME mass. Alternatively, a different approach is also possible by subtracting the $24 \mathrm{hr}$ minimum background image (Figure 10, bottom left), in which each pixel has the 

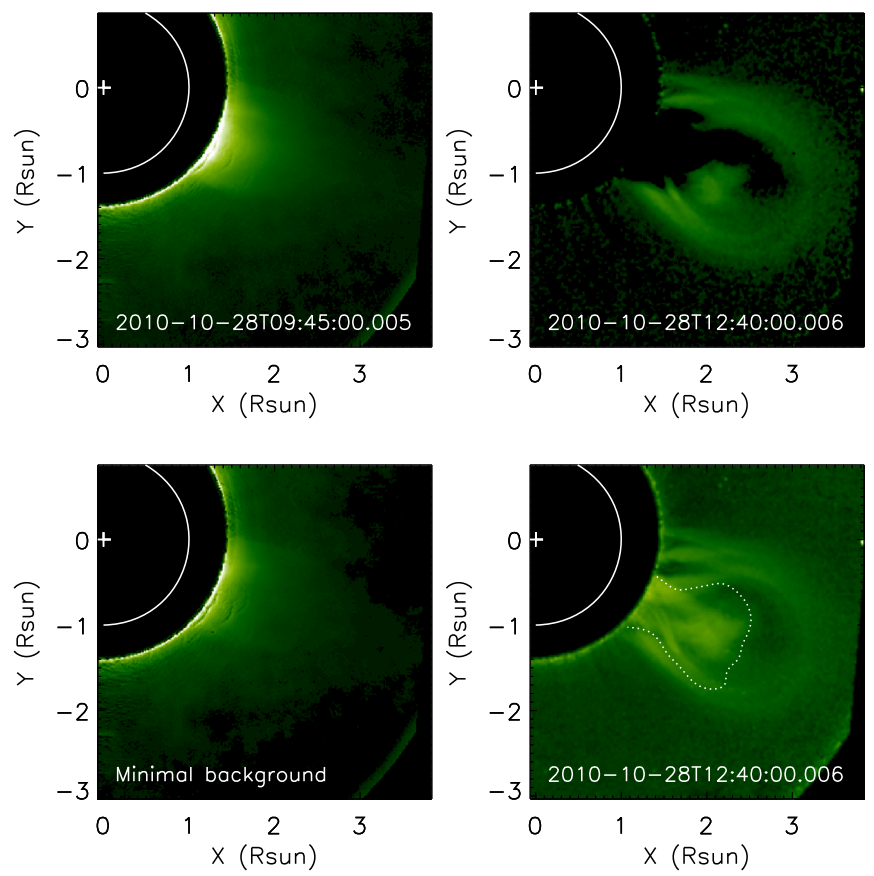

Figure 10. Left column: comparison between two different background preevent images. The upper panel is the preevent background image acquired at $\sim 09: 45$ UT, while the lower panel is the $24 \mathrm{hr}$ minimum background image. Right column: base-difference images at $\sim 12: 40$ UT obtained by subtracting the two different background images shown in the left column. The dotted line represents the region of the CME core, which is defined in Section 3.4.

minimal brightness observed from $\sim 00: 00$ UT to $\sim 23: 55$ UT; in this way we obtained the new difference image shown in Figure 10 (bottom right). This image, containing some streamer structure, can be then converted to the mass image and be regarded as the upper limit of the CME mass. The mass distributions of the $\mathrm{CME}$, derived from the $\mathrm{pB}$ images via the SolarSoft routine el theory . pro, are presented in Figure 11. Here we derived two different mass distributions by subtracting the two different background images mentioned above. The region of interest we consider to estimate the CME mass corresponds to the region where we measured the radial velocity, while the remaining part is ignored here. Then we can calculate the potential energy distribution of the CME, hence the energy required to lift the CME plasma from the solar surface to its position $R$, according to the equation

$$
E_{p}=\int_{R_{\odot}}^{R} \frac{G M_{\odot} m_{\mathrm{CME}}}{r^{2}} d r(\mathrm{erg}),
$$

where $G$ is the gravitational constant, $M_{\odot}$ is the solar mass, $m_{\mathrm{CME}}$ is the mass of the CME plasma, and $R$ is the radial distance from the solar center. The potential energy of the CME at $\sim 12: 40$ UT is shown in Figure 11 (bottom panels).

On the other hand, the CME kinetic energy distribution is given by

$$
E_{k}=\frac{1}{2} m_{\mathrm{CME}} v^{2}(\mathrm{erg}),
$$

where, by indicating with $v_{\theta}$ the plasma velocity along the latitudinal direction in the POS, and with $v_{\phi}$ the plasma velocity along the longitudinal direction, the total velocity $v$ is given by $v=\sqrt{v_{r}^{2}+v_{\theta}^{2}+v_{\phi}^{2}}$. As we mentioned in Section 3.1, this
CME was almost propagating in the POS, so the measured radial velocity in the POS can be used as an approximate to its $3 \mathrm{D}$ radial speed $v_{r}$ in space. Unfortunately, we cannot estimate the latitudinal and longitudinal velocities directly from our method. Here we choose two ways to estimate the total kinetic energy of the CME: (1) by only considering the radial velocity; (2) by measuring the tangential speed of the CME plasma with latitudinal slices in the VL images (such as the dotted line shown in Figure 6) and by assuming that the longitudinal velocity of the CME plasma is equal to the measured latitudinal velocity. By using the polar coordinate data mentioned above, it is easy to make slices along the latitude at the same radial altitude; in particular we use a width of the slices of $0.125 R_{\odot}$. For example, to analyze this CME at 12:40 UT, we make around 20 latitudinal slices and obtain time-distance plots at different positions. Subsequently, by comparing all velocity results, we acquire the maximal latitudinal speed of the CME flank and regard it as the average speed of a packet of plasma in each pixel cell, by assuming an equality between the longitudinal and latitudinal speeds. The kinetic energy will be overestimated when the latitudinal speed distribution should be inhomogeneous and small.

Finally, the derived kinetic energy distributions of the CME body are shown in Figure 12. Here, we choose the average velocity obtained from the AS procedure, multiplying with the different mass distributions of the CME shown in Figure 11, to estimate the kinetic energy and mechanical energy of the CME at 12:40 UT. The total kinetic energy is $\sim(3.18-10.7) \times 10^{29} \mathrm{erg}$, and the average of the total kinetic energy is $6.94 \times 10^{29} \mathrm{erg}$. Actually, when we just calculate the kinetic energy by the radial velocity, the result is only $7 \%$ less than the kinetic energy with all of the three components of velocity taken into account.

We obtain the CME's mechanical energy, $E_{M}$, combining its potential and total kinetic energy. The evolution of total $E_{M}, E_{k}$, and $E_{p}$ from 11:25 UT to 12:50 UT is shown in Figure 13. Notice that during this period the structure of the CME, still in the FOV of STEREO A/COR1, can be distinguished and used to measure its velocities with our method. In Figure 13 (top panel), the total mechanical energy of the CME enhances around from $1.7 \times 10^{30}$ to $3.9 \times 10^{30} \mathrm{erg}$ with a stable energy increasing rate of $4.3 \times 10^{26} \mathrm{erg} \mathrm{s}^{-1}$; the blue dots represent the average mechanical energy, while the blue shadow indicates uncertainties propagated from the mass measurements. For this CME, due to its low-speed movement, the kinetic energy is far smaller than its potential energy, as shown in Figure 13 (bottom panel). The increase of the kinetic energy is due to both the mass and the speed increase. The dots and shadow regions also represent the average value and error of potential and kinetic energy in blue and red (pink) color, respectively. Vourlidas et al. (2000) analyzed the energy budget of $11 \mathrm{CMEs}$ and found that the kinetic energy is smaller than the potential energy for relatively small CMEs, but larger for fast CMEs $\left(v_{\mathrm{cme}} \geqslant 600 \mathrm{~km} \mathrm{~s}^{-1}\right)$. Due to the conservation of the total energy (consisting of the kinetic, potential, and magnetic energies and neglecting other energy forms such as thermal energy and wave energy), the investigators thought that this result indicates that slow CMEs are magnetically driven, a conclusion that is reinforced by the small magnitude of the plasma $\beta$ parameter. 

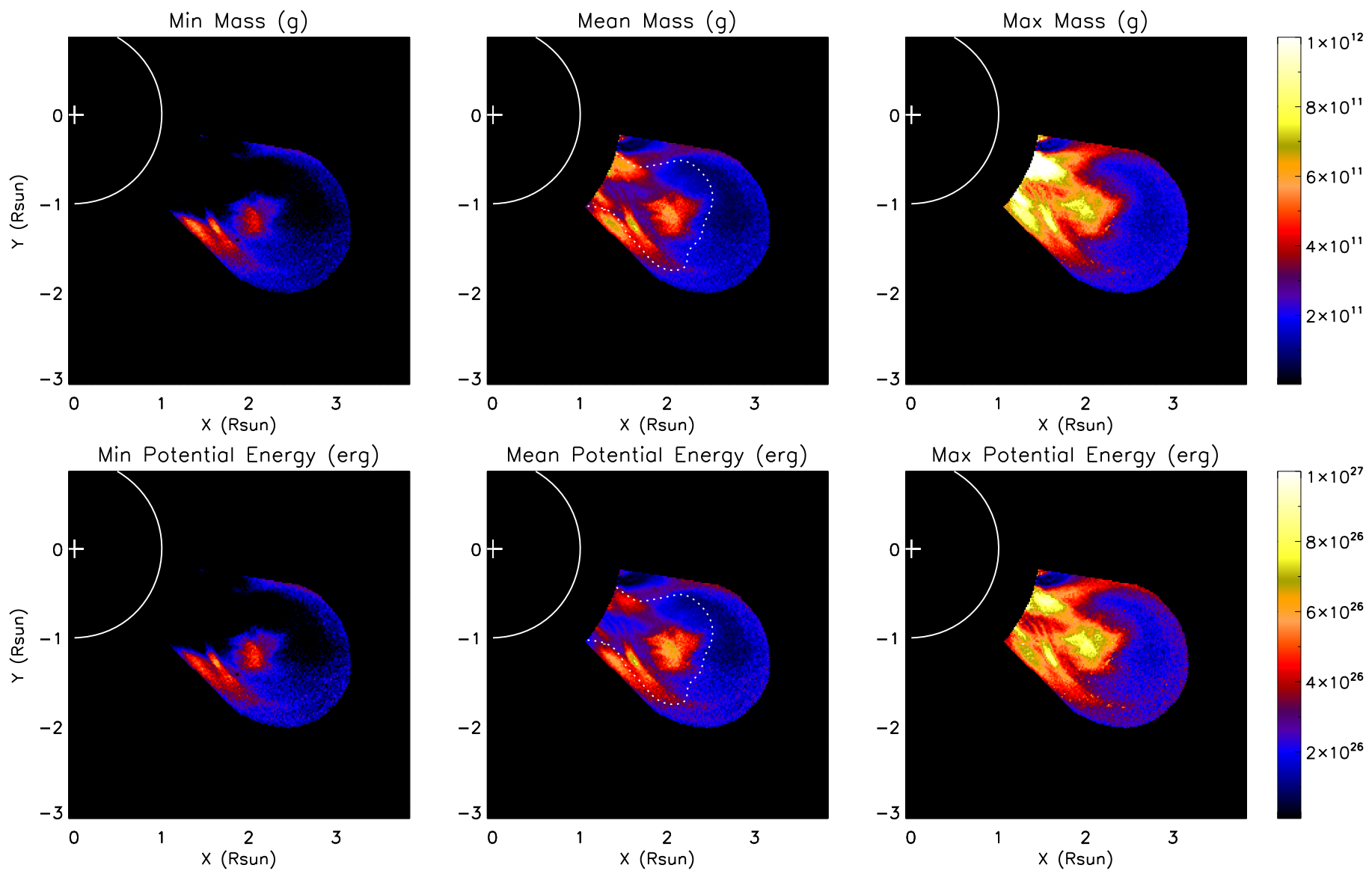

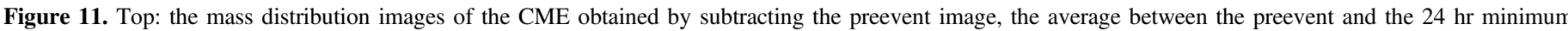

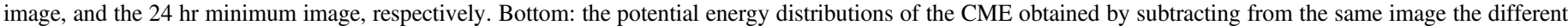
background images shown in the top panels. The dotted lines represent the region of the CME core.

It is well-known that a typical CME has a three-part structure, consisting of (1) a bright leading edge, (2) a dark cavity, and (3) a bright dense core (Illing \& Hundhausen 1985), originating from the explosive magnetic flux rope system. Many investigators believe that the dark void and the bright core correspond to the whole flux rope and the magnetic dips of the flux rope (e.g., Low \& Hundhausen 1995; Chen 1996; Gibson \& Fan 2006; Cheng et al. 2012), respectively. Cheng et al. (2012) has analyzed the density and temperature of the CME leading front and revealed that the CME bright front results from the compression of ambient plasma when the CME expands its core region. Thus, in our work, we also discuss the energy distribution of the CME's core and front. In order to define these two "core" and "front" regions in a more quantitative way, we follow this procedure: for each VL base-difference image subtracting the $24 \mathrm{hr}$ minimum background, we define "by hand" a region of interest where the core should be located, taking this region to be as big as possible. Then, in this region, we identify the maximum intensity $I_{\max }$ and define as pixels belonging to the CME core those with an intensity between 1 and a fraction $f$ of $I_{\max }$ (optimizing the value of $f$, here $f=0.07)$. Then, once we have identified all the pixels belonging to the core in this way, we assume that all the rest of the CME region belongs to the "front+void" instead. In this way we make a separation between the CME "core" on one hand and the CME "front+void" on the other hand, as was done by Howard (2015a, 2015b). The CME core is marked by the dotted line in Figure 10 (bottom right).
The relationship between the mass of the CME and the calculated centroid distance is shown in Figure 14 (top panel). In Figure 14 the blue signs indicate the mass evolution of the CME core, which is almost constant with the centroid distance (or with the time), while the mass of the CME front, marked by the pink symbols, increases with its propagation toward the interplanetary space. The centroid distance is calculated by $r_{c}=\frac{\sum m_{i} r_{i}}{\sum m_{i}}$, where $m_{i}$ and $r_{i}$ denote the mass and distance from Sun center, respectively, of each pixel. The uncertainties shown here are propagated from the CME mass measurements with two different background subtractions. This result shows that during the CME propagation a significant mass is being added mostly at the CME front and not at the core, and this is in agreement with the fact that the core should be more "magnetically isolated" from the rest of the CME because it is surrounded by the flux rope, while the front, on the one hand, being made also by coronal plasma dragged up during the CME propagation, is expected to have a mass increase, which also has been reported by many investigators (Vourlidas et al. 2000; Cheng et al. 2012; Howard 2015b). The same result was already demonstrated with the analysis of $\mathrm{SOHO} / \mathrm{UVCS}$ data, deriving the elemental distribution of CME plasma embedded in the front compared with the plasma in the surrounding corona (Ciaravella et al. 2003). But on the other hand, Feng et al. (2015b) used a "snow plow" model to calculate the mass contribution of the pileup solar wind and found that the effect of the solar wind pileup is not sufficient to explain the observed 

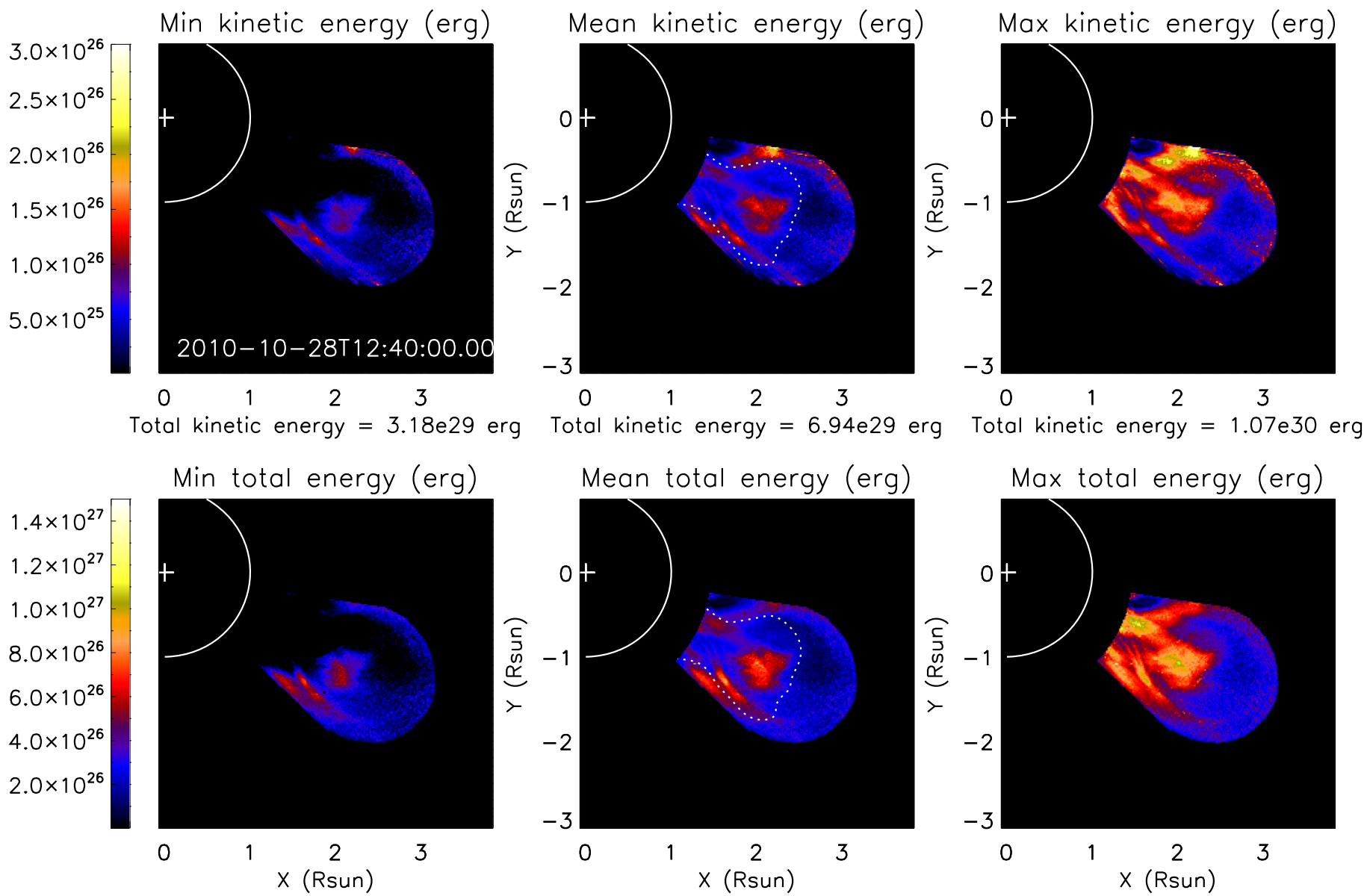

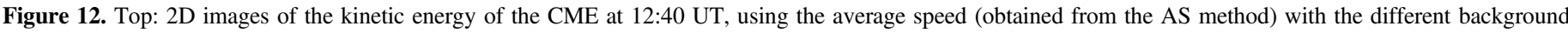

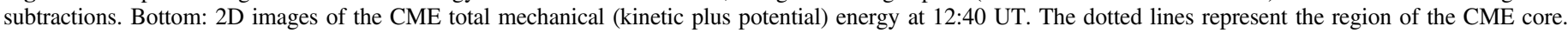

mass increase. Many investigators think the increase of the CME mass is mainly due to the mass supplement by the outflow from dimming regions in the low corona (Aschwanden et al. 2009; Jin et al. 2009; Tian et al. 2012). What's more, Howard \& Vourlidas (2018) studied the evolution of the volume electron density of $13 \mathrm{CME}$ fronts and found that there is no obvious pileup observed in the front region of the CMEs from 2.6 to $30 R_{\odot}$. Whether the mass increase outside the core region of CME mostly comes from the solar wind pileup or the dimming region outflow requires further analysis and is beyond the scope of this paper.

In Figure 14 (bottom panel), the red and blue dots represent the average mechanical energy of the CME core and front, respectively, while the shadows indicate the upper and lower limits of the mechanical energy due to the mass measurement with different background subtractions. Figure 14 shows that at the initial stage of the CME, the mechanical energy of the core is higher than that of the front. Then, with the propagation of the CME, the energy of the front rises more rapidly, reaching up to that of the core. This is likely an indirect consequence of the CME formation: at the early stages the eruption is driven by the expansion of the CME flux rope, thus containing the larger fraction of mechanical energy; then, as the front plasma is dragged by the expanding flux rope and progressively accelerated, its mechanical energy progressively increases with time, reaching a final stage where both core and front contain approximately the same amount of mechanical energy.

\section{Discussion and Conclusions}

The purpose of our work is to measure the radial velocity distribution of the CME plasma in the POS. The radial velocity distribution of the CME plasma can provide important information, while combining the VL and UV channels coronagraph observations. These observations will be provided in the next few years by the Metis coronagraph on board Solar Orbiter and by the LST coronagraph on board the ASO-S. The results presented here will be really useful for the diagnostics of the temperature distribution of CMEs, in combination with the temperature diagnostics discussed by Bemporad et al. (2018). In this work, we develop a new method based on the crosscorrelation method to measure the $2 \mathrm{D}$ radial speed distribution of the CME body through synthetic VL coronagraphic images from the MHD simulation. Tappin et al. (1999) only applied this method to calculate the outflow speeds of the solar wind without considering the variations of speeds at different position angles (and structures) in the images. Comparing with the actual radial velocity from the MHD simulation, we can estimate the measured error and thus apply this method to a real event. The main results are listed below:

1. We successfully use the synthetic VL images to measure the radial velocity distribution of the CME in the POS. Using two kinds of $2 \mathrm{D}$ radial reference velocities of the $\mathrm{CME}$ obtained from the MHD simulation (one is integrated along the LOS and averaged with density, the other one is the cut of radial velocity in the POS), we compare the measured radial 

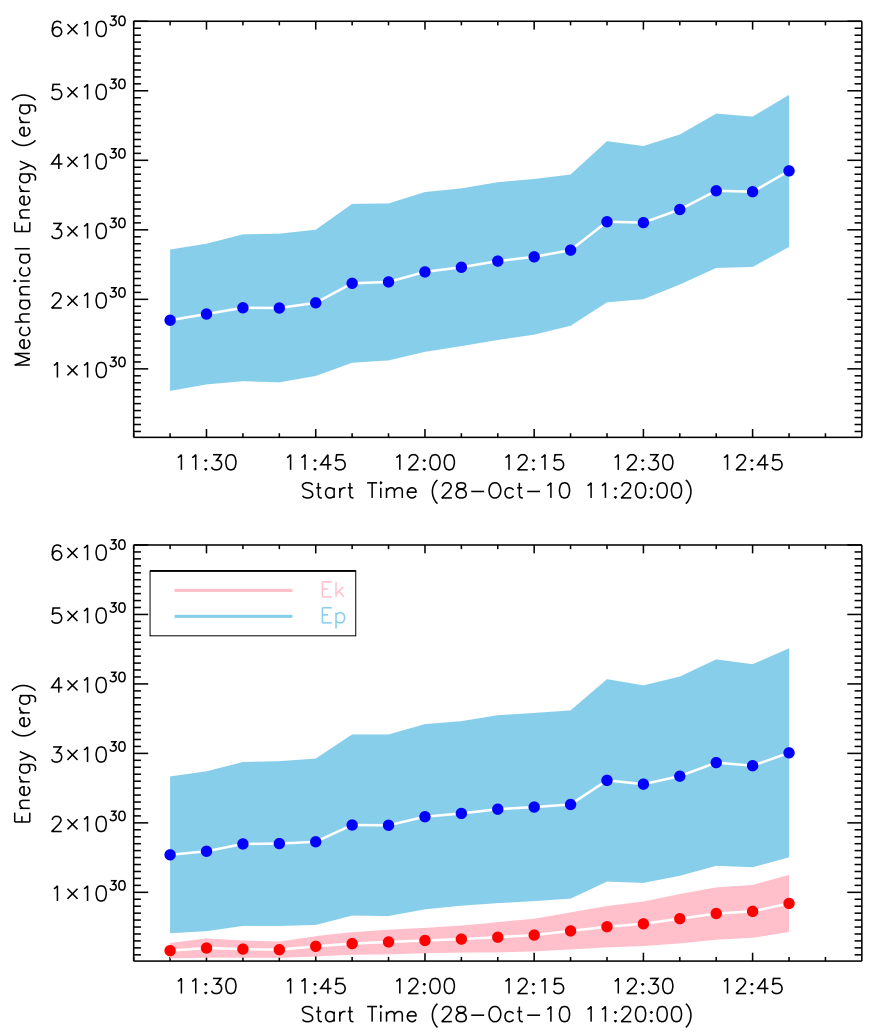

Figure 13. Top: evolution of the mechanical energy of the CME with the time. Bottom: evolution of the kinetic and potential energy of the CME with time. Pink (blue) region is the range of the kinetic (potential) energy. Red (blue) dots show the average value of the kinetic (potential) energy.

velocity with these two reference velocities and estimate the measured error between these two velocity distributions. The result shows that the measured velocity obtained from the synthetic VL images is well matched with the reference velocity integrated along the LOS and averaged with the electron number density in the core with no more than $20 \%$ uncertainty, while it is matched better with the simulated radial velocity in the POS in the front within $30 \%$ uncertainty. On the other hand large uncertainties are present in the CME's flanks.

2. We apply the same method to a real CME event observed by STEREO A/COR1 propagating with a low speed of $\sim 320 \mathrm{~km} \mathrm{~s}^{-1}$ at the nose. The 2D radial velocity distribution of this CME shows the anisotropic kinematic feature of the CME plasma when this CME is propagating along the POS. The major velocity of the CME body is slower than the speed of the CME nose, while some parts, especially in the CME flank, have a higher speed, which is still less than $\sim 400 \mathrm{~km} \mathrm{~s}^{-1}$.

3. Owing to the low speed of the CME, here we could estimate the 2D distribution of the Doppler dimming factors for the UV HI Ly $\alpha$ spectral line, because this factor is really sensitive to the radial component of outflow velocity. If the plasma speed is larger than $300 \mathrm{~km} \mathrm{~s}^{-1}$, this factor is almost zero. This character of the Doppler dimming factor indirectly confirms that (as recently shown by Bemporad et al. 2018) the UV intensity in the CME front will be severely attenuated due to the larger plasma radial speed.

4. We derived for the first time the 2D kinetic energy distribution with a three-component structure under the assumption that longitudinal speed is equal to the latitudinal speed, while the latitudinal speed is measured by the slices tracked in the VL images. We also discuss the $2 \mathrm{D}$ mechanical energy distribution
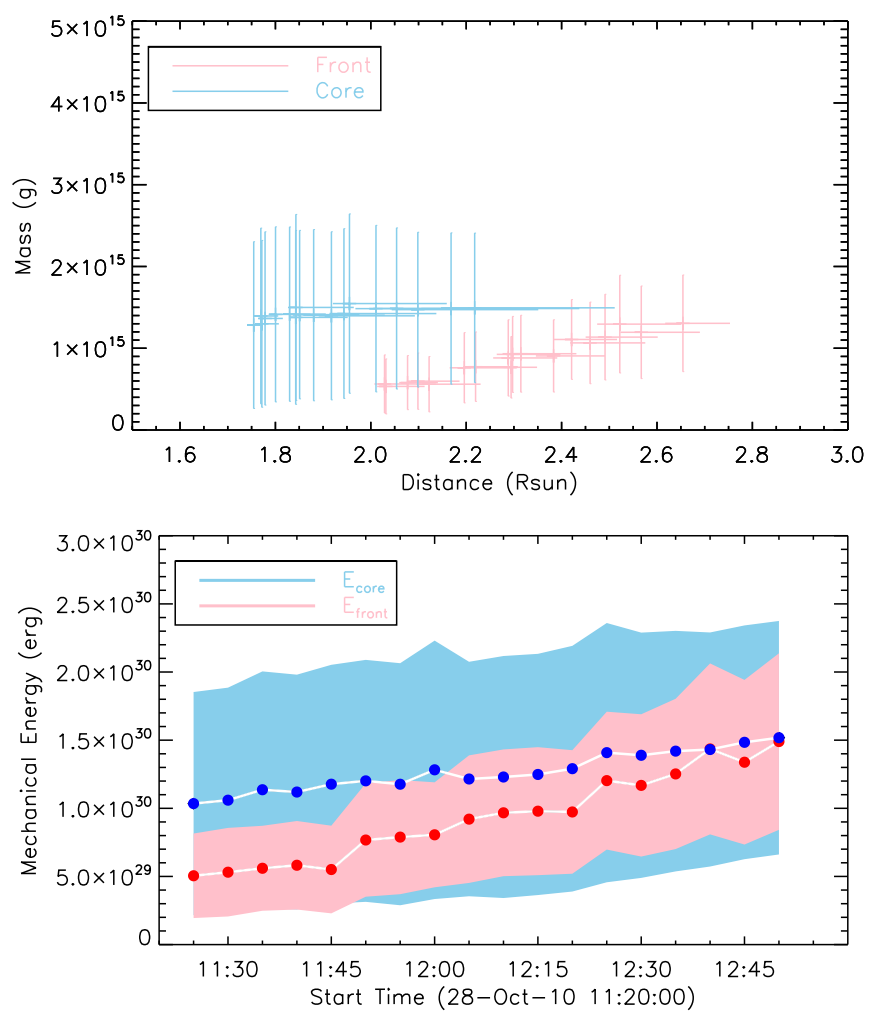

Figure 14. Top: mass vs. centroid distance measurements of the core (blue) and its front (pink) components of the CME. Error bars are estimated by subtracting the different backgrounds. Bottom: evolution of the mechanical energy measured from the CME core (blue shadow) and front (pink shadow) parts. Blue and red dots represent the average mechanical energy of the CME core and front, respectively. The shadows show the error ranges of the mechanical energy.

and time evolution (combining the kinetic and potential energies) in the different parts (core and front) of the CME. For this lowspeed CME, the potential of the CME is 1 order of magnitude higher than the kinetic energy. The increase of the kinetic energy is due to both the mass and the speed increase. In the early phases the mechanical energy of the CME core is larger than that of the CME front; then, with time evolution, the front's energy reaches up to the energy of the core. This could be a signature of the CME formation processes, if the CME is originally driven by the expansion of the flux rope leading to subsequent acceleration of plasma embedded in the front.

5. During the CME propagation, the mass of the core almost keeps constant, while that of the CME front increases with the rising of the CME. This is consistent with the classical picture for the formation of the three-part structure of the CME: the dark cavity corresponds to the whole flux rope, and the core, which is more magnetically isolated, does not significantly increase its mass during the CME expansion, while the front forms by the mass supplement from the dimming region in the low corona and/or pileup of the ambient plasma, leading to a progressive mass increase.

In this work, we compared the difference between the kinetic energy with and without considering the latitudinal and longitudinal speed of the CME. The comparison ratio between these two kinds of kinetic energies is no more than $10 \%$ during the evolution of the CME from 11:25 to 12:50 UT. Given the mechanical energy evolution and distribution of the CME core and front, the impact made by these two additional kinetic 
energy components is thus negligible, because the energy difference between these two kinetic energies is no more than $2 \%$ of the total mechanical energy. The largest uncertainty of the energy is related to the estimate of the CME mass, which was performed here with two different background subtractions, providing upper and lower limit estimates of the CME mass. This is important because MHD simulations (Lugaz et al. 2005) and other works dealing with the CME mass measurement (e.g., Vourlidas et al. 2000, 2010; Howard 2015b) have shown that the estimate of the CME masses based on the Thomson-scattering method may be underestimated by a factor of $\sim 2$.

Doppler dimming factor $F_{D}$ plays a significant role in calculating the radiative emission due to the resonant scattering of the chromospheric $\mathrm{HI} \mathrm{Ly} \alpha$ emission by very few remaining neutral hydrogen atoms in the hot corona. This parameter will be affected by the radial velocity of the plasma, the normalized coronal absorption profile, and the intensity spectrum of the incident profile, as mentioned above. In our work, we do not consider the velocity projection effect due to the propagation of the real CME along the POS. It is possible to correct the projection effect via the polarization-ratio technique mentioned by Susino \& Bemporad (2016). The purpose of our work is to provide a good tool to derive the $2 \mathrm{D}$ radial velocity distribution of the CME, so that in the future we can combine the VL and UV coronagraphic observations, thanks to the future Solar Orbiter/ Metis and ASO-S/LST instruments, to diagnose the characteristics of the CMEs, such as the kinetic energy distribution, temperature distribution, and so on. Bemporad et al. (2018) tested three different hypotheses to estimate the CME plasma temperatures by taking the Doppler dimming factor into account: (1) full collisional excitation assumption, (2) full radiative excitation assumption, and (3) radiative and collisional excitation assumption. The results in Bemporad et al. (2018) showed that all temperatures estimated by the synthetic VL and UV images were underestimated, whatever method is considered to take into account LOS integration effects.

As we all know, the Sun is variable, constantly evolving in time. For the UV intensity observations from the future coronagraphs, whether there are ARs or not in the solar disk, will lead to an immeasurable different intensity measurement of the resonant radiative contributions from the chromosphere. In the era of $A S O-S$, LST will be the first supply the full-disk observation of the $\mathrm{H} \mathrm{I} \mathrm{Ly} \alpha$ line, which can allow us to constrain the incident radiation intensity from the chromosphere layer and enhance the accuracy of the calculation of the Doppler dimming factor, subsequently decreasing the uncertainty of the CME temperature estimation.

A good approach to understanding the dynamical evolution of the physical parameters of the erupting $\mathrm{CME}$ is to analyze the CME energy budget. The energy of the CME propagating outward consists of the kinetic, potential, magnetic, and other energy forms. Many investigators have found that there should exist additional heating sources to heat CMEs, to reproduce the plasma temperatures as derived from UV emission and taking into account the CME plasma cooling mainly due to the adiabatic expansion. The corresponding thermal energy can be comparable or even higher than the kinetic and potential energies carried by the CMEs (Akmal et al. 2001; Lee et al. 2009; Landi et al. 2010; Murphy et al. 2011). Furthermore, the temperature increase of the plasma during the early CME expansion phase also implied the existence of an additional thermal energy source
(Bemporad et al. 2007). If CMEs are assumed to be a perfect gas in local thermodynamic equilibrium with equal electron and ion temperatures, the enthalpy can be as large as five times thermal energy. The upper limit of the enthalpy of a normal CME will be 1 order of magnitude less than the kinetic and potential energies, except in the lower corona, where it can be comparable to the kinetic energy (Vourlidas et al. 2000). For fast CMEs, the magnetic energy is negligible compared with the potential and kinetic energy, while slow CMEs could be driven by magnetic force. Hence, the estimate of the 2D temperature distribution inside CMEs will reveal the real evolution of the CME thermal energy during their expansion. The total energies of the CMEs are comparable to the range of flare energies estimated from nonthermal electrons (Aschwanden \& Parnell 2002; Emslie et al. 2004, 2005; Feng et al. 2013). In other words, eruptive events possibly share a relationship of an energy-equal partition between CMEs and flares. Furthermore, combining with the analysis of the energy partition between the flares and CMEs among the various components would provide valuable constraints on the fundamental energy release process(es) (Reeves et al. 2010; Reeves \& Moats 2010), thus helping us to understand the origin of CMEs.

The authors thank Dr. Thomas Wiegelmann and Dr. Bernd Inhester at the Max-Planck-Institut for Solar System Research for laying the foundation for the preparation of the article. STEREO is a project of NASA. The SECCHI data used here were produced by an international consortium of the Naval Research Laboratory (USA), Lockheed Martin Solar and Astrophysics Lab (USA), NASA Goddard Space Flight Center (USA), Rutherford Appleton Laboratory (UK), University of Birmingham (UK), Max-Planck-Institut for Solar System Research (Germany), Centre Spatiale de Liège (Belgium), Institut d'Optique Théorique et Appliqueé (France), and Institut d'Astrophysique Spatiale (France). This work is supported by NSFC (grant Nos. 11522328, 11473070, 11427803, and U1731241), by CAS Strategic Pioneer Program on Space Science (grant Nos. XDA15010600, XDA15052200, XDA15320103, and XDA15320301), and by the National Key R\&D Program of China (2018YFA0404200). This research has received funding from the European Union Horizon 2020 research and innovation program (grant agreement No. 647214). This work used the DiRAC@Durham facility managed by the Institute for Computational Cosmology on behalf of the STFC DiRAC HPC Facility (www.dirac.ac.uk). The equipment was funded by BEIS capital funding via STFC capital grants ST/P002293/1, ST/R002371/1, and ST/S002502/1, Durham University, and STFC operations grant ST/R000832/1. DiRAC is part of the National e-Infrastructure. We acknowledge the use of the open source (gitorious.org/amrvac) MPI-AMRVAC software, relying on coding efforts by C. Xia, O. Porth, and R. Keppens.

\section{ORCID iDs}

Beili Ying (i) https://orcid.org/0000-0001-8402-9748 Alessandro Bemporad (ib https://orcid.org/0000-00015796-5653

Silvio Giordano (ib https://orcid.org/0000-0002-3468-8566

Paolo Pagano (iD https://orcid.org/0000-0001-5274-515X

Li Feng (1) https://orcid.org/0000-0003-4655-6939

Lei Lu (10) https://orcid.org/0000-0002-3032-6066

Hui Li (ib https://orcid.org/0000-0003-1078-3021 


\section{References}

Akmal, A., Raymond, J. C., Vourlidas, A., et al. 2001, ApJ, 553, 922 Antonucci, E., Andretta, V., Cesare, S., et al. 2017, Proc. SPIE, 10566, 105660L

Aschwanden, M. J. 2016, ApJ, 831, 105

Aschwanden, M. J., Nitta, N. V., Wuelser, J.-P., et al. 2009, ApJ, 706, 376

Aschwanden, M. J., \& Parnell, C. E. 2002, ApJ, 572, 1048

Bemporad, A., Pagano, P., \& Giordano, S. 2018, A\&A, 619, A25

Bemporad, A., Raymond, J., Poletto, G., \& Romoli, M. 2007, ApJ, 655, 576

Billings, D. E. 1966, A guide to the solar corona (New York: Academic Press)

Braga, C. R., Dal Lago, A., Echer, E., Stenborg, G., \& Rodrigues Souza de Mendonça, R. 2017, ApJ, 842, 134

Brueckner, G. E., Howard, R. a., Koomen, M. J., et al. 1995, SoPh, 162, 357

Carley, E. P., McAteer, R. T. J., \& Gallagher, P. T. 2012, ApJ, 752, 36

Chen, J. 1996, JGR, 101, 27499

Cheng, X., Zhang, J., Saar, S. H., \& Ding, M. D. 2012, ApJ, 761, 62

Ciaravella, A., Raymond, J. C., \& Kahler, S. W. 2006, ApJ, 652, 774

Ciaravella, A., Raymond, J. C., van Ballegooijen, A., et al. 2003, ApJ, 597, 1118

Colaninno, R. C., \& Vourlidas, A. 2006, ApJ, 652, 1747

Colaninno, R. C., \& Vourlidas, A. 2009, ApJ, 698, 852

Emslie, A. G., Dennis, B. R., Holman, G. D., \& Hudson, H. S. 2005, JGRA, 110, A11103

Emslie, A. G., Kucharek, H., Dennis, B. R., et al. 2004, JGRA, 109, A10104

Feng, L., Inhester, B., \& Gan, W. 2015a, ApJ, 805, 113

Feng, L., Inhester, B., Wei, Y., et al. 2012, ApJ, 751, 18

Feng, L., Li, H., Chen, B., et al. 2019, RAA, in press

Feng, L., Wang, Y., Shen, F., et al. 2015b, ApJ, 812, 70

Feng, L., Wiegelmann, T., Su, Y., et al. 2013, ApJ, 765, 37

Gan, W., Deng, Y., Li, H., et al. 2015, Proc. SPIE, 9604, 96040T

Gibson, S. E., \& Fan, Y. 2006, JGRA, 111, A12103

Gopalswamy, N., Yashiro, S., Michalek, G., et al. 2009, EM\&P, 104, 295

Howard, R. A., \& Vourlidas, A. 2018, SoPh, 293, 55

Howard, T. A. 2015a, ApJ, 806, 175
Howard, T. A. 2015b, ApJ, 806, 176

Illing, R. M. E., \& Hundhausen, A. J. 1985, JGR, 90, 275

Jin, M., Ding, M. D., Chen, P. F., Fang, C., \& Imada, S. 2009, ApJ, 702, 27

Kaiser, M. L., Kucera, T. A., Davila, J. M., et al. 2008, SSRv, 136, 5

Kohl, J. L., Esser, R., Gardner, L. D., et al. 1995, SoPh, 162, 313

Landi, E., Raymond, J. C., Miralles, M. P., \& Hara, H. 2010, ApJ, 711, 75

Lee, J.-Y., Raymond, J. C., Ko, Y.-K., \& Kim, K.-S. 2009, ApJ, 692, 1271

Lemaire, P., Emerich, C., Vial, J.-C., et al. 2002, in ESA Special Publication, 508, From Solar Min to Max: Half a Solar Cycle with SOHO, ed. A. Wilson (Noordwijk: ESA Publications), 219

Li, H. 2015, in Proc. IAU 320, Solar and Stellar Flares and their Effects on Planets, ed. A. G. Kosovichev, S. L. Hawley, \& P. Heinzel, 436

Li, H., Chen, B., Feng, L., et al. 2019, RAA, in press

Low, B. C., \& Hundhausen, A. J. 1987, JGRA, 92, 2221

Low, B. C., \& Hundhausen, J. R. 1995, ApJ, 443, 818

Lu, L., Inhester, B., Feng, L., Liu, S., \& Zhao, X. 2017, ApJ, 835, 188

Lugaz, N., Manchester, W. B., IV, \& Gombosi, T. I. 2005, ApJ, 627, 1019

Mierla, M., Chifu, I., Inhester, B., Rodriguez, L., \& Zhukov, A. 2011, A\&A, 530, L1

Murphy, N. A., Raymond, J. C., \& Korreck, K. E. 2011, ApJ, 735, 17

Noci, G., Kohl, J. L., \& Withbroe, G. L. 1987, ApJ, 315, 706

Pagano, P., Mackay, D. H., \& Poedts, S. 2014, A\&A, 568, A120

Raymond, J. C., Ciaravella, A., Dobrzycka, D., et al. 2003, ApJ, 597, 1106

Reeves, K. K., Linker, J. A., Mikić, Z., \& Forbes, T. G. 2010, ApJ, 72 1547

Reeves, K. K., \& Moats, S. J. 2010, ApJ, 712, 429

Robbrecht, E., \& Berghmans, D. 2004, A\&A, 425, 1097

Susino, R., \& Bemporad, A. 2016, ApJ, 830, 58

Tappin, S. J., Simnett, G. M., \& Lyons, M. A. 1999, A\&A, 350, 302

Thernisien, A., Vourlidas, A., \& Howard, R. A. 2009, SoPh, 256, 111

Thernisien, A. F. R., Howard, R. A., \& Vourlidas, A. 2006, ApJ, 652, 763

Tian, H., McIntosh, S. W., Xia, L., He, J., \& Wang, X. 2012, ApJ, 748, 106

Vourlidas, A., Howard, R. A., Esfandiari, E., et al. 2010, ApJ, 722, 1522

Vourlidas, A., Subramanian, P., Dere, K. P., \& Howard, R. A. 2000, ApJ, 534,456 